\title{
Preparation of the Essential Oil from Artemisia Argyi Grown in Qichun, China and its application in Antibacterial effection
}

\author{
Hui Hu${ }^{1}$, Qingan $\mathrm{Li}^{1}$, Shenxi Chen ${ }^{1}$, Yuancai Liu ${ }^{1}$, Huameng Gong ${ }^{1}$, Bukun Jin ${ }^{1 *}$ \\ ${ }^{1}$ Jing Brand BIO-Medicine Co., Ltd, Huangshi 435100, China
}

\begin{abstract}
To evaluate the antibacterial activity and chemical constituents of the essential oil from the artemisia argyi grown in Qichun (China). METHODS: Steam distillation method was used to extract volatile oil from Artemisia argyi. The antibacterial effect of the volatile oil was investigated by the plate coating method and the double gradient liquid dilution method. Gas chromatography-mass spectrometry(GC-MS) was applied for the identification of chemical constituents in volatile oil from Artemisia argyi and the relative percentage of each component was calculated by area normalization. RESULTS: The essential oil from artemisia argyi grown in Qichun (China) has significant antibacterial activity against staphylococcus aureus, pseudomonas aeruginosa, salmonella, candida albicans, aspergillus niger and aspergillus flavus. And fifty chemical components were detected in the essential oil, and twenty compounds were identified, accounting for $95.95 \%$ of total essential oil. And the artemisol in artemisia argyi grown in Qichun (China) was found to be the highest compared with the same species from other producing areas. CONCLUSION: The essential oil from artemisia argyi grown in Qichun (China) was a potent antibacterial plant extract with potential applications as an antibacterial drugs or food preservative.
\end{abstract}

\section{Introduction}

Essential oils are present in various aromatic plants that are commonly grown in tropical and subtropical countries. Several techniques have been used to obtain essential oils from the plant, including steam distillation, solvent extraction, cold pressing and supercritical fluid extraction. ${ }^{[1]}$ Among these techniques, essential oils are most commonly obtained by a steam distillation method. The major constituents of essential oils are terpenes, terpenoids and aromatic and aliphatic compounds, which are characterized as low-molecular-weight aroma chemicals. ${ }^{[2-4]}$ The essential oils are extremely permeable to the skin and entering the body through the abundant capillaries under subcutaneous fat attributed to the relatively short molecular chains of essential oils. Nowadays, essential oils and some of their components have been widely used in various products, including cosmetics, household cleaning products and air fresheners, hygiene products, agriculture, and food, as well as in medicinal uses. ${ }^{[5-7]}$

Artemisia argyi is widely distributed throughout China, which is one of the most popular plants in Chinese traditional medicine and frequently used for diseases treatment such as eczema, inflammation, hemostasis, menstruation-related symptoms, and tuberculosis. Recent study on Artemisia argyi found that both its alcohol and water extracts exhibited good antibacterial, [8] analgesic and anti-inflammatory, [9] antifatigue, [10] immune regulation ${ }^{[11]}$ and antitumor effects. Extensive studies on the chemical constituents of
Plant material and essential oil extractionartemisia argyi have shown that many constituents are identified from the dried leaves, such as monoterpenes, sesquiterpenes and triterpenes. ${ }^{[12-15]}$

Artemisia argyi have strong aroma with complicated volatile components. The volatile components of artemisia argyi from different origins may have obvious differences due to the differences of environment and climatic conditions. Xiao Yushuo et al. analyzed the volatile oil constituents of 13 batches of artemisia argyi in different producing areas, and determined the main components which affecting the volatile quality evaluation of artemisia argyi by common peak principal component analysis. and then these two principal components were selected to analysis the volatile quality of each batch of artemisia argyi (Table 1). The higher the total factor score $\mathrm{F}$, the better the quality of the volatile oil in the batch. As shown in Table 1, the total factor score of the volatile oil from the leaves of were significantly higher, and the quality of the volatile oil was better than that of the other leaves. Therefore, we selected the artemisia argyi from Qichun for further research. The artemisia argyi from Qichun is called treasures due to its widely applications in medicine. Modern researches indicate that volatile oils, flavonoids, tannins and organic acids are the main chemical components and functional ingredients of artemisia argyi from Qichun.The aim of the present study was to assess the antibacterial activity of the essential oil obtained from artemisia argyi grown in Qichun, China. 


\section{Materials and methods}

\subsection{Plant material and essential oil extraction}

Artemisia argyi were collected during May in Qichun, China. Three samples of the leaves (2 $\mathrm{kg}$ for each samples) from artemisia argyi were hydrodistilled for $5 \mathrm{~h}$ in extraction equipment. The essential oil was collected for further analysis.

\subsection{Activation of the test strains}

Each test strain was inoculated into a liquid medium from a test tube, cultured at $37^{\circ} \mathrm{C}$ for $18 \mathrm{~h}$, and then streaked on a plate to obtain a pure activated strain.

\subsection{Preparation of test bacterial solution}

Bacterial solution: The activated purebred bacteria was inoculated in liquid medium, incubated at $37^{\circ} \mathrm{C}$ for $18 \mathrm{~h}$, and adjusted the bacterial count to $103-104 \mathrm{CFU} / \mathrm{mL}$ with physiological saline after the tablet counts for further use. Fungal spore suspension: The activated purebred fungus was inoculated into a plate and cultured at $30{ }^{\circ} \mathrm{C}$ for $2-3 \mathrm{~d}$. After the dense spores appeared on the plate, the spores were eluted with physiological saline. The eluate was then placed in a triangular flask with glass beads and shaken on a shaker to break up the spores in the eluate. Finally, a spore suspension of aspergillus niger and aspergillus flavus were obtained by filtering through four layers of gauze. However, Candida albicans is a yeast, and the preparation of its bacterial solution is the same as that of bacteria.

\subsection{Preparation of test samples of different concentrations}

The essential oil of artemisia argyi was emulsified with $2 \%$ Tween-80 to prepare $200 \mu \mathrm{L} / \mathrm{mL}$ essential oil emulsion for further use. The different concentrations of essential oil $(100 \mu \mathrm{L} / \mathrm{mL}, 50 \mu \mathrm{L} / \mathrm{mL}, 25 \mu \mathrm{L} / \mathrm{mL}, 12.5$ $\mu \mathrm{L} / \mathrm{mL}, 6.25 \mu \mathrm{L} / \mathrm{mL}, 3.12 \mu \mathrm{L} / \mathrm{mL}$ ) were prepared by double gradient liquid dilution method under sterile conditions, and labeled as sample No. 1-6.

\subsection{Determination of minimum inhibitory concentration (MIC)}

The minimum inhibitory concentration (MIC) was determined by plate coating method and the main steps were as follows. Pipette $0.1 \mathrm{~mL}$ of bacterial solution or spore suspension with $0.1 \mathrm{~mL}$ of different concentrations of 1-6 samples solution. After the solution was evenly mixed, it was applied to LB solid medium or PDA medium, and placed in a sterile operation table for a period of time. After the medium was completely absorbed, the mixture was placed into a $37^{\circ} \mathrm{C}$ incubator for 1-2 $\mathrm{d}$ and then the experimental results could be observed. At the same time, the positive control and negative control were set.

\section{Results and discussion}

Table 1. Principal component factor scores of the volatile oil constituents from artemisia argyi leaves of different origins.

\begin{tabular}{ccccc}
\hline & Batches & $\mathrm{F} 1$ & $\mathrm{~F} 2$ & $\mathrm{~F}$ \\
\hline Nantong, & 1 & 0.125 & -0.094 & 0.026 \\
Jiangsu & 2 & 0.124 & -0.095 & 0.025 \\
\hline & 3 & 1.220 & -0.099 & 0.622 \\
Qichun, & 4 & 1.400 & -0.053 & 0.741 \\
Hubei & 5 & 1.430 & -0.04 & 0.764 \\
& 6 & 0.600 & 0.154 & 0.398 \\
& 7 & 0.710 & 0.145 & 0.454 \\
\hline Taizhou, & 8 & 0.125 & -0.093 & 0.026 \\
Zhejiang & 9 & 0.071 & 0.151 & 0.107 \\
\hline Chuxiong, & 10 & 0.072 & 0.153 & 0.109 \\
Yunnan & 11 & 0.137 & -0.002 & 0.074 \\
\hline Wulumuqi, & 12 & 0.063 & 0.152 & 0.103 \\
Xinjiang & 13 & 0.056 & 0.152 & 0.100 \\
\hline
\end{tabular}




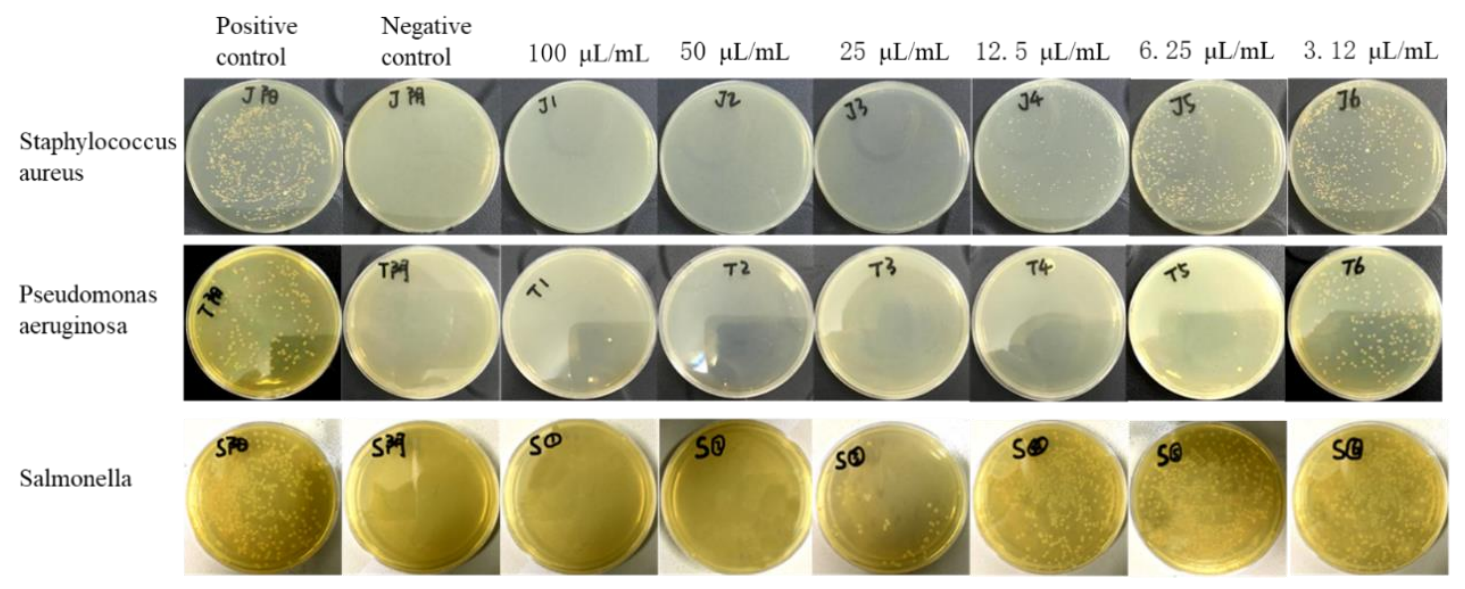

Fig. 1 The MIC of the volatile oil from artemisia argyi against the pathogenic bacteria.

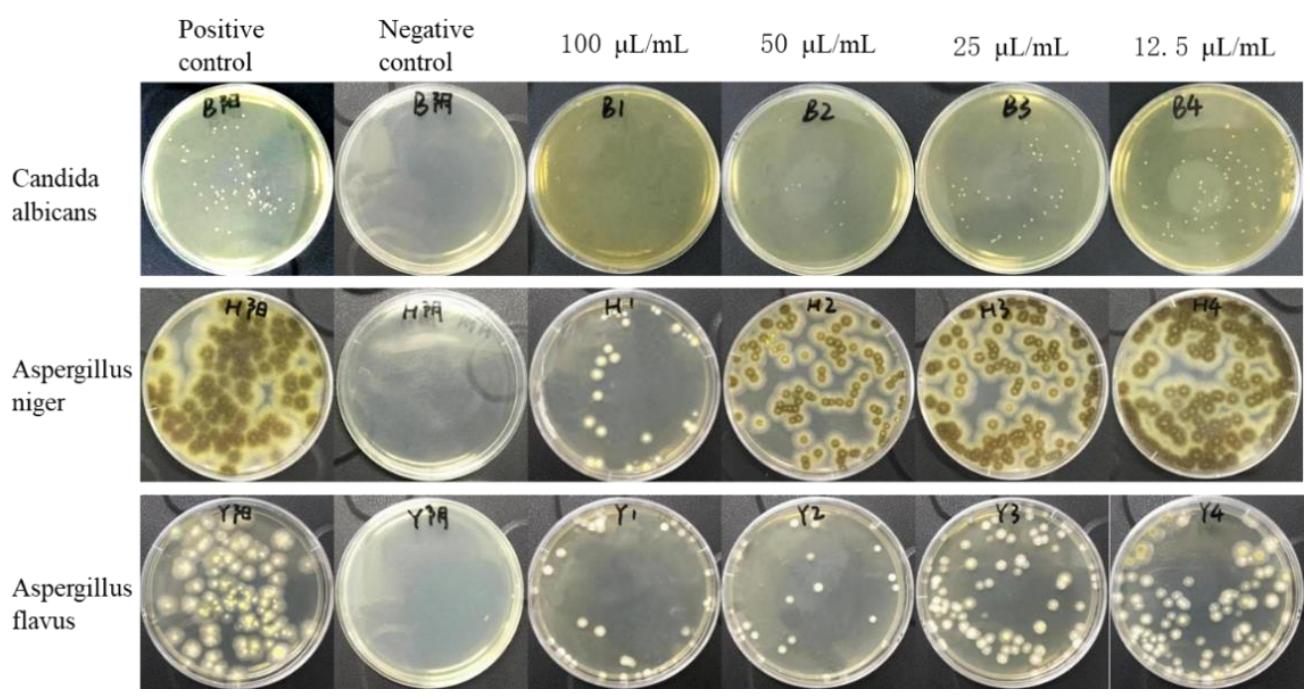

Fig.2 The MIC of the volatile oil from artemisia argyi against the fungi.

As shown in Fig.1, the volatile oil from artemisia argyi had obvious antibacterial effect on the above bacteria compared with the positive control, group and the MIC of it against staphylococcus aureus, pseudomonas aeruginosa and salmonella were $25 \mu \mathrm{L} / \mathrm{mL}, 6.25 \mu \mathrm{L} / \mathrm{mL}$ and $50 \mu \mathrm{L} / \mathrm{mL}$, respectively.

As shown in Fig. 2, the aspergillus niger and aspergillus flavus had a large and numerous colony and longer hyphae on the positive control plate. Meanwhile, the aspergillus niger and aspergillus flavus producing black spores and yellow spores, respectively. It can be clearly seen that when the concentration of the drug solution at $100 \mu \mathrm{L} / \mathrm{mL}$, the colonies of aspergillus niger on the plate were smaller and less, the hyphae were shorter, and there was no black spores. It indicating that the $80-90 \%$ of aspergillus niger was sufficiently inhibited. So the MIC of the volatile oil from artemisia argyi against aspergillus niger was $100 \mu \mathrm{L} / \mathrm{mL}$. Similarly, when the concentration of the drug solution was greater than or equal to $50 \mu \mathrm{L} / \mathrm{mL}$, the $80-90 \%$ of aspergillus flavus was sufficiently suppressed to growth. And with the decrease of the concentration of the drug solution, the growth of aspergillus flavus on the plate was basically the same as that of the positive control group, so the MIC of the volatile oil from artemisia argyi against aspergillus flavus was $50 \mu \mathrm{L} / \mathrm{mL}$.

\section{Conclution}

The results of MIC of the essential oil against staphylococcus aureus, pseudomonas aeruginosa and salmonella indicated that the volatile oil from artemisia argyi had significant antibacterial effect on the tested bacteria which could provide a basis for applying in the clinical treatment of bacterial infections.

And the results of MIC of the volatile oil from artemisia argyi against candida albicans, aspergillus 
niger and aspergillus flavus indicating that the volatile oil had significant inhibition effect on the test fungi. Based on this property, the volatile oil from artemisia argyi might have great prospects for applying in antifungal treatment which caused by candida albicans. On the other hand, aspergillus niger and aspergillus flavus were widely exist in food, thus the volatile oil from artemisia argyi would be a promising food preservatives in the future.

\section{Acknowledgement}

This research was financially supported by Hubei Province Technology Innovation Special Project (No. 2018ACA125).

\section{References}

1. Shaaban HAE, El-Ghorab AH and Shibamoto T. Bioactivity of essential oils and their volatile aroma components: Review. Journal of Essential Oil Research, 24, 203-212 (2012).

2. Tsibranska I, Tylkowski B, Kochanov R, Alipieva K. Extraction of biologically active compounds from Sideritis ssp. L. Food \& Bioproducts Processing , 89, 273-280 (2011).

3. Betts TJ. Chemical characterisation of the different types of volatile oil constituents 3by various solute retention ratios with the use of conventional and novel commercial gas chromatographic stationary phases. J. Chromatogr. A, 936, 33-46 (2001).

4. Pichersky E, Noel JP and Dudareva N. Biosynthesis of plant volatiles: Nature's diversity and ingenuity. Science ; 311, 808-811 (2006).

5. Silva J, Abebe W, Sousa SM, Duarte VG, Machado MIL and Matos FJA. Analgesic and antiinflammatory effects of essential oils of Eucalyptus. Journal of Ethnopharmacology , 89, 277-283(2003).

6. Hajhashemi V, Ghannadi A and Sharif B. Antiinflammatory and analgesic properties of the leaf extracts and essential oil of Lavandula angustifolia Mill. Journal of Ethnopharmacol , 89: 67-71 (2003).
7. Perry NS, Bollen C, Perry EK and Ballard C. Salvia for dementia therapy: Review of pharmacological activity and pilot tolerability clinical trial. Pharmacol. Biochem. Behav , 75, 651-659 (2003).

8. Chen Y, Xu W and Xu SY. Germicidal Effect of the Leaves of Artemisia argyi Extract. Journal of Microbiology, 34, 107-109 (2014).

9. Jiang H, Hou AJ, Xiang ZX and Chen YX. Study on Artemisia Argyi Essential Oil :Anti-inflammatory, Antianaphylatic and Analgesic Effects. New medical journal, 15, 36-39 (2005).

10. Jiang H, Hou AJ, Xiang ZX and Chen YX. AntiFatigue Effect of Essential Oil Extracted from Artemisia Argyi. Medical Journal of Wuhan University, 26,373-374 (2005).

11. Huang J, Chen YX, Hou AJ, Jiang H and Duan HP. Immunomodulatory Effects of Essential Oil Extracted from Artemisia Argyi on Mice. Pharmacology and Clinical of Chinese Medicine ,21,21-22(2005).

12. Jeong MA, Lee KW, Yoon DY and Lee HJ. Jaceosidin, a pharmacologically active flavone derived from, inhibits phorbolester-induced upregulation of COX-2 and MMP-9 by blocking 12phosphorylation of ERK-1 and -2 in cultured human mammary epithelial cells. Ann. NY Acad. Sci. 1095, 458-466 (2007).

13. Kim MJ, Kim DH, Lee KW, Yoon DY and Surh YJ. Jaceosidin induces apoptosis in ras-transformed human breast epithelial cells through generation of reactive oxygen species. Ann. NY Acad. Sci. 1095, 483-495 (2007).

14. Lan MB, Zhang YH and Zheng Y. Antioxidant and Immunomodulatory Activities of Polysaccharides from Moxa (Artemisia argyi) Leaf. Food Sci. Biotechnol. 19,1463-1469 (2010).

15. Seo JM, Kang HM, Son KH, Kim JH, Lee CW, Kim $\mathrm{HM}$, et al. Antitumor activity of flavones isolated from Artemisia argyi. Planta Med. 69, 218-222 (2003) 